\title{
Determination of active ingredients in commercial insecticides using spectral characteristics of Fourier transform infrared spectroscopy (FTIR)
}

\section{B. Asan Mohamed}

Department of Soil Science and Agricultural Chemistry, Tamil Nadu Agricultural University, Coimbatore - 641003 (Tamil Nadu), India.

\section{P. Janaki*}

Department of Soil Science and Agricultural Chemistry, Tamil Nadu Agricultural University, Coimbatore - 641003 (Tamil Nadu), India.

*Corresponding author. Email: janaki.p@tnau.ac.in

\section{Article Info}

https://doi.org/10.31018/ jans.v13iSI.2809

Received: March 22, 2021

Revised: May 14, 2021

Accepted: June 3, 2021

\section{How to Cite}

Asan Mohamed, B. and Janaki, P. (2021). Determination of active ingredients in commercial insecticides using spectral characteristics of Fourier transform infrared spectroscopy (FTIR). Journal of Applied and Natural Science, 13 (SI), 110 - 123. https://doi.org/10.31018/jans.v13iSI.2809

\begin{abstract}
Pesticides have become a basic necessity for yield development. This might be credited to the quickly expanding population, which has presented weight on the food creation industry.Fourier Transform Infra-red Spectroscopy utilizes sample with less course of action, less time consuming, simple, fast, non-destructive and environmental friendly infrared-based method. It makes use of Smart iTR window and pellets use on omnic transmission window. In FTIR the peaks formed for the representative sample are from $800 \mathrm{~cm}^{-1}$ to $4000 \mathrm{~cm}^{-1}$ of wavenumbers against the \% transmittance. The FTIR spectra obtained for pesticide formulations were on par with the NIST (National Institute of Standards and Technology) spectra library. Comparing the commercial-grade spectra with the Spectrabase, NIST library and Bio-rad software showed the peak ranges for different functional groups of the compound and can be examined with KnowltAll software's ProcessltIR and AnalyseltIR. We can obtain the active principle of the peak, peak intensities. This method can be viewed as genuine choices to long and tedious chromatographic strategies as a rule suggested for quality control of commercially accessible pesticide formulations and check for adultered formulations that harm agricultural produce.
\end{abstract}

Keywords: FT-IR, Functional groups, Pesticides, Quality control, Wave numbers

\section{INTRODUCTION}

Agriculture is the world's major conservative movement, with over half of the total population being reliant upon agriculture for their livelihood. Pesticides empower the amounts and the nature of yields and food to be controlled and restrict the numerous human illnesses transmitted by insect or rodent vectors. In any case, despite their numerous benefits, pesticides are the absolute generally poisonous, residual and versatile substances in nature. Their unnecessary use deleteriously affects people and nature; their essence in food is especially hazardous. With their ecological security, the capacity to bio-accumulate and harmfulness, pesticides may put the human body in more danger of illness and harm (Fenik et al., 2011).

The enrollment, assembling and offer of a pesticide plan infer various controls among which its assess- ment, security and creation are the most significant. To portray a pesticide, it is important to have the option to decide its composition and chemical and physical properties. The main advantages of Near Infra-Red spectrometry are its nondestructive nature, the possibility to analyze products in real-time, the low cost of equipment maintenance, the fast response times and the possibility to measure directly solid samples, with no sample pre-treatment(Moros et al., 2006). Chromatographic methods have been the most broadly utilized techniques today. Regardless, the dynamic standards of the samples permit us that vibrational spectrometrybased systems could be utilized as a genuine option in the quality control of commercial pesticide formulations. Commercially available pesticides are being availed through different chemical formulations such as granular, wettable powder, liquid formulations etc. As the determination of these pesticides entails special extrac- 
tion procedures which demand costly solvents, time consumable procedures and some other technical aspects to ascertain. By using FTIR spectroscopy, the easy, effective and short time determination of commercial-grade pesticide formulations can be carried out without any processing of samples like in GC-MS, LCMS, etc. (Armenta et al., 2007)

Fourier transform infrared (FTIR) is a fast and microdestructive spectroscopy method widely applied in the measurement of solid (Post et al., 1995), liquid (Van de Voort et al., 2004), and gas (Esler et al., 2000). This technique is used to identify the compounds' functional groups through different strategies, and FTIR works at a different range of spectra. For the identification of spectra of pesticides active ingredients, mostly the ATR -FTIR is used at mid-range. The connection between ATR-FTIR spectroscopy and other comparative strategies, of all the things considered known as vibrational spectroscopy (Lee et al., 2017).

FTIR method was found advantageous for Folpet and Metalaxyl, respectively, found comparable with liquid chromatography results with UV detection and involves a considerable decrease in solvent consumption (Quintas et al., 2003). Cyromazine determined with FTIR and FT-Raman procedure were found statistically compared with reference liquid chromatography procedure and showed the FTIR methods were appropriate for quality control in commercial pesticide formulations (Armenta et al., 2004). The waste generation in FT-IR for diuron determination was $3.4 \mathrm{ml} \mathrm{CHCl}_{3}$, in flow injection analysis $9.3 \mathrm{ml} \mathrm{CHCl}_{3}$ per sample and those methods consume less organic solvent than an HPLC method, which involves the use of $39 \mathrm{~mL}$ of acetonitrile per sample (Armenta et al., 2005b). By utilizing FTIR spectrometry, the immediate assurance of malathion in commercial pesticide formulations can be completed, with no pre-treatment of tests, with reproducibility and precision practically identical to those measured utilizing GC -FID, besides, decreasedtime and volume of chlorinated solvents utilized (from $35 \mathrm{ml}$ of $\mathrm{CHCl}_{3}$ to $2 \mathrm{ml}$ ) in the analysis (Quintás et al., 2004b).

The most well-known practice in the direct examination of solids by IR spectrometry is the utilization of disks arranged from the samples mixed in dry $\mathrm{KBr}$. This method stays away from the utilization of any sort of dissolvable and does not require the analyte to be soluble. Be that as it may, it makes inconvenience for the assurance of the bandpass and for the most part, requires the utilization of an internal standard. That is the purpose behind the limited quantity of papers found in writing utilizing direct estimations on $\mathrm{KBr}$ plates (Armenta et al., 2005c)

To stimulate the determination of pesticides active ingredients with easy processing methods, less time consuming, environmentally friendly and reduced costeffective techniques need to be recognized and adopt- ed. Using the spectral characteristics to analyze the active ingredient in commercial pesticides is the best alternative to the ransom and time-consuming chromatography technique. Hence the present study was performed to investigate the applicability of FTIR technique and get the spectral region of sensitivity for the quick determination of active ingredient in various commercially available pesticides at Tiruchirappalli.

\section{MATERIALS AND METHODS}

\section{Commercial grade chemicals used}

Commercially available pesticides belonging to 21 groups/classes viz., abamectin, ketonenols, neonicotinoid, organochlorine, organofluorine, organophosphates, phenylpyrazole, pyrethroids, quinazoline and thiourea were analysed in FTIR to identify the active ingredients. The powdered and liquid formulations of pesticides were analyzed using transmission window and ATR-Diamond window, respectively. Spectra obtained for each pesticide formulation was processed using Bio-rad software and also compared with the Spectrabase and NIST library. The formulations utilized in the study are presented in Table 1.

\section{Fourier transform infrared spectroscopy (FT-IR)}

Fourier transform infrared spectroscopy (FT-IR) is a technique which is used to obtain an infrared spectrum of absorption, emission, photoconductivity or Raman scattering of a solid, liquid or gas. An FTIR spectrometer simultaneously collects spectral data in a wide spectral range. The instrument FTIR spectrometry used was Nicolet iS10 using OMNIC spectra software. Two windows were used for sample analysis, such as SmartITR window and Omnic transmission window. Here the Spectral data were collected by a Bio-Rad Excalibur 3000 MX FTIR spectrometer and a helium-purged MTEC 300 photoacoustic cell. All the spectra were recorded over the $4000-400 \mathrm{~cm}^{-1}$ region at a spectral resolution of $8 \mathrm{~cm}^{-1}$ and with the 1024 scans co-added. The $\mathrm{KBr}$ was used as a pelleting material with powdered and granule formulations and liquid formulations were directly fed in the iTR window.

\section{FTIR procedure}

The details of the conditions under which the spectra of insecticides formulations obtained are presented in Table 2.

\section{Omnictransmission window}

The powder formulations of insecticides were compressed into a thin pellet for analyzes by FTIR. For the preparation of pesticide pellet samples, IR transparent material, namely $\mathrm{KBr}$ was mixed at the ratio of $2: 1$ in a mortar and pestle for 5 - 10 minutes. Then the mixture was converted into pellets by pressing the prepared 


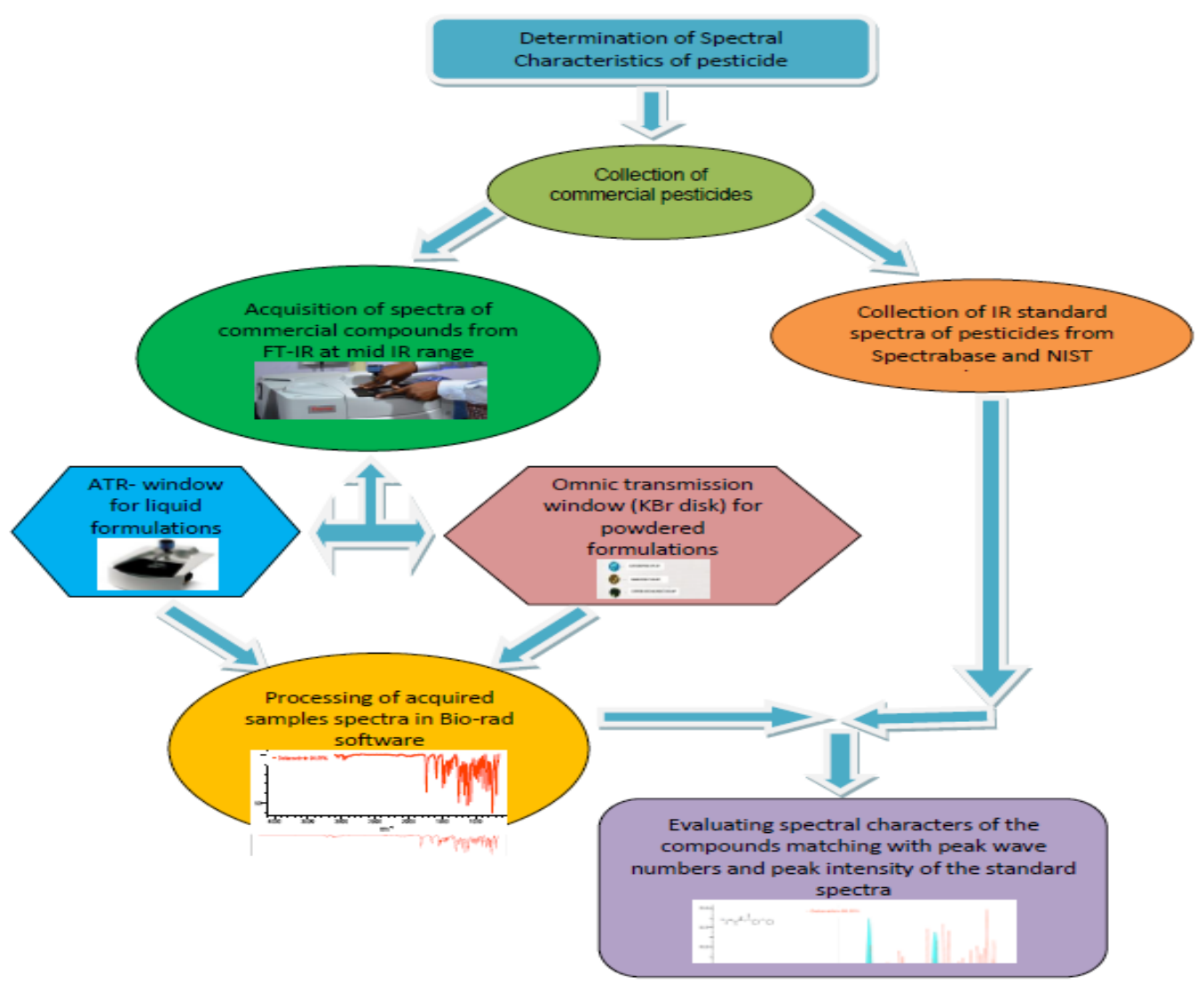

Fig. 1. Flow Chart of the determination procedure.

mixture with a hydraulic or hand press into a hard disk. A total laboratory hydraulic press creating a power (force) around 15 tons was used to make a pellet of ideally 0.5 to $1 \mathrm{~mm}$ thick, which was then placed in a transmission holder and scanned.

\section{Attenuated total reflectance window}

ATR can be used to analyze free-flowing aqueous solutions, viscous liquids, coatings, ecological materials. This technique is often the preferred method for liquid analysis because it simply requires a drop of liquid to be placed on the crystal. In the present study, the ATR window was used for the analysis of liquid pesticide formulations using the Diamond platform. A drop of the compound was placed on the platform in which the infra-red light was present and locked with the screw. Software Omni was used to get the spectrum which was compiled with instrument provides the compound spectrum within 30 to 40 seconds of their intact. It provides the spectrum in absorbance, transmission and other properties.

\section{Processing and comparison of sample spectra with database}

The sample spectra obtained from FTIR were compared with the NIST library and processed using the Bio-Rad KnowltAll software. The NIST (National Insti- tute of Standards and Technology) provides Standard Reference Data, which spread a wide scope of logical orders including nuclear and sub-atomic material science, synthetic and precious stone structures, liquids, material properties, biotechnology, optical character acknowledgement and more. SpectraBase is a free online spectral vault from Bio-Rad Laboratories, Inc. The sample spectra can be processed through a huge number of spectra, including natural mixes, inorganic mixes, and polymers. The KnowltAll programming offers far-reaching answers for IR, Raman, NIR, NMR, MS, UV-Vis, and chromatography the flow chart of the determination procedure were given in Fig. 1. The product joined with the world's biggest spectral library, enables scientific experts to separate significantly more prominent information from their phantom information. The results of sample spectrabase processed are presented and discussed here.

\section{RESULTS AND DISCUSSION}

Results obtained are presented and discussed below.

\section{Abamectin}

It was observed that Emamectin 5\% SG contained amines and alcohols as functional groups and have $\mathrm{NH}, \mathrm{NH}_{2}$, and $\mathrm{NH}_{3}$ salts. The analyzedSpectra(Fig. 2a) 
showed bands at $1340-1250 \mathrm{~cm}^{-1}$ by medium symmetric primary amine $\mathrm{NH}_{3}$ salt, secondary amine salt of $\mathrm{NH}$ at $2800-2000 \mathrm{~cm}^{-1}$ and $\mathrm{NH}_{2}$ at $850-750 \mathrm{~cm}^{-1}$. Alcohol group showed strong stretching of hydrogen-bonded $\mathrm{OH}$ at $3300-3280 \mathrm{~cm}^{-1}$. It has $\mathrm{NH}_{2}$ peak intensity of $69.76 \%$ at $759.82 \mathrm{~cm}^{-1}$ (Fig. 2b).

\section{Ketoenols}

Spiromesifen derives from a 1,3,5-trimethylbenzene and a 3,3-dimethylbutyric acid. The commercial compound spectrum(Fig. 3a) examined with the bio-rad's know-it-all software showed the peak ranges shown in Fig. 3b. Spiromesifen spectrum had absorption bands at 2959-2952 and $2866-2853 \mathrm{~cm}^{-1}$ due to asymmetric and symmetric stretching of $\mathrm{CH}$ bond in cyclopentyl group. It contained $\mathrm{C}=\mathrm{C}$ bond variable stretching of alkane $\mathrm{CCCH}=\mathrm{CHCC}$ groups at $1680-1620 \mathrm{~cm}^{-1}$. The intensity of the peak at $1022.09 \mathrm{~cm}^{-1}$ showed $100 \%$ in ProcessItIR.

\section{Neonicotinoids}

Acetamiprid is a $\mathrm{N}$-[(6-chloropyridin-3-yl) methyl]-N'cyano-N-methylethanimidamide. FTIR analysis results of acetamiprid $20 \%$ SP (Fig.4a) showed the presence of $\mathrm{C}-\mathrm{Cl}$ bond stretching at $830-600 \mathrm{~cm}^{-1}$. The amine bonds of $\mathrm{NH}_{2}$ and $\mathrm{NH}$ was located at 1620-1560, 850750 and $2800-2000 \mathrm{~cm}^{-1}(\mathrm{NH})$. The peak at 1531.34 $\mathrm{cm}^{-1}$ due to $\mathrm{N}-\mathrm{H}$ in plane deformation has $100 \%$ intensity which was very strong and characteristic of $\mathrm{N}$ atom attached to $\mathrm{C}$ atom of aromatic ring. The out-of-plane $\mathrm{N}$ -H deformation vibration at $900-600 \mathrm{~cm}^{-1}$ was masked by absorption features of aromatic ring(Fig. 4b).

Imidacloprid is a systemic insecticide utilized as a foliar spray for the control of different sucking pests and other important bugs of cotton, paddy, chillies, sugarcane, sunflower, okra and mango. FT-IR spectra (fig. 5a) showed strong $\mathrm{CH}$ stretching at $2800-2900 \mathrm{~cm}^{-1}$ and $2690-2775 \mathrm{~cm}^{-1}$. The signature bands(fig.5b) of $1667-$ $1680,1650-1550$ and $830-600 \mathrm{~cm}^{-1}$ due to strong stretching of $\mathrm{C}=\mathrm{O}$ bond, nitrile group $(\mathrm{C}-\mathrm{N}=\mathrm{C})$ and halogen group $\mathrm{C}-\mathrm{Cl}$ was seen in the spectra. The peak intensity is $100 \%$ at $1667.64 \mathrm{~cm}^{-1}$ and $32.03 \%$ at $1566.88 \mathrm{~cm}^{-1}$. Quintás et al. (2004a) reported $-\mathrm{NO}_{2}$ symmetric stretching band at $1300-1250 \mathrm{~cm}^{-1}$.

Thiacloprid is a member of thiazolidines, a nitrile and a monochloropyridine. It derives from a 2-chlorop yridine and a cyanamide. The Thiacloprid $21.7 \%$ SC spectrum (Fig.6a) examined in the KnowltAll software (Fig.6b) showed the presence of nitrile group $\mathrm{C}-\mathrm{N}=\mathrm{C}$ with strong stretched vibration $\mathrm{C}=\mathrm{N}$ bond at $1550-1650$ $\mathrm{cm}^{-1}$. Spectrum also had strong stretching of halogen $\mathrm{C}$ $-\mathrm{Cl}$ bond at $600-830 \mathrm{~cm}^{-1}$. The peak intensity observed with the spectrum showed $68.79 \%$ intensity at 1627.63 $\mathrm{cm}^{-1}$ and $100 \%$ at $652.59 \mathrm{~cm}^{-1}$ and confirmed the presence of nitrile and halogen functional groups.

Thiamethoxam is a xenobiotic and a neonicotinoid insecticide. It is an oxadiazane, a member of 1,3- thiazoles, an organochlorine compound and a 2nitroguanidine derivative. Thiamethoxam 30\% FS spectrum (Fig.7a)examined with the bio-rad's software (Fig.7b)showed medium stretching of $\mathrm{C}-\mathrm{N}$ bond amine group at $1310-1360 \mathrm{~cm}^{-1}$ and strong stretching of $\mathrm{C}-\mathrm{Cl}$ bond halogen group at $600-830 \mathrm{~cm}^{-1}$. The observed peak intensity of $99.99 \%$ at $652.59 \mathrm{~cm}^{-1}$ and $46.13 \%$ at $1634.86 \mathrm{~cm}^{-1}$ showed the halogen and nitrile bonds vibration.

\section{Organochlorines}

Dicofol is a nonflowable fluid (or waxy strong), extending from dim to yellow-earthy color and is a viable acaricide controls mites, spider parasites on different yields. Dicofol 18.5\% EC (Fig.8a) spectrum processed with ProcessltIR (Fig.8b) of the bio-rad software showed strong stretching bond of halogen group $\mathrm{C}-\mathrm{Cl}$ in $830-600 \mathrm{~cm}^{-1}$. It also had bands at $3400-3200 \mathrm{~cm}^{-1}$ and $1480-1410 \mathrm{~cm}^{-1}$ due to strong stretching and deformation of $\mathrm{OH}$ bond of $(\mathrm{R})_{2} \mathrm{CH}-\mathrm{OH}$, respectively. It showed the $100 \%$ intensity of $\mathrm{C}=\mathrm{O}$ group stretching at $1094.41 \mathrm{~cm}^{-1}$. Results are equated with the NIST spectrum.

\section{Organofluorines}

Flubendiamide is $1-N$-[4-(1,1,1,2,3,3,3-heptafluoropro pan-2-yl)-2-methylphenyl]-3-iodo-2-N-(2-methyl-1methylsulfonylpropan-2-yl)benzene-1,2-dicarboxamide. The commercial formulations of $39.35 \%$ SC (Fig.9a) spectrum showed the presence of the $\mathrm{P}$ group, sulphur compounds and amine groups. The $\mathrm{NH}$ bond occurred in the range of $2320-2700 \mathrm{~cm}^{-1}, \mathrm{PH}_{2}$ bond stretching in the range of $2271-2440 \mathrm{~cm}^{-1}$ and sulphur bonds like $\mathrm{SO}_{2}, \mathrm{~S}-\mathrm{O}$ and S-C occurs at 1342-1352 $\mathrm{cm}^{-1}, 891-910$ $\mathrm{cm}^{-1}$ and $600-700 \mathrm{~cm}^{-1}$ (Fig.9b).

\section{Organophosphates}

Acephatebelongs to methamidophos is a mixed diacylamine, a phosphoramide, an organic thiophosphate and an organothiophosphate insecticide in which one of the hydrogen is replaced by an acetyl group. Acephate raw sample spectrum (Fig.10a) obtained from FTIR showed $\mathrm{C}=\mathrm{O}$ stretching at $1697.36 \mathrm{~cm}^{-1}$, and sharp and strong absorption of $\mathrm{P}=\mathrm{O}$ stretching frequency at $1219.71 \mathrm{~cm}^{-1}$ and were attributed to the presence of $\mathrm{C}=\mathrm{O}$ and $\mathrm{P}=\mathrm{O}$ bonds in the structure. As compared to the positions of the bonds in the spectrum of acephate, the presence of $\mathrm{C}=\mathrm{O}$ (carbonyl) and $\mathrm{P}=\mathrm{O}$ groups was confirmed in the structure of acephate. Fig.10e showed that the bands at $1697.05 \mathrm{~cm}^{-1}$ and $1034.14 \mathrm{~cm}^{-1}$ had an intensity of 32.98 and $100.00 \%$ was obtained using Processlt IR. The present results were comparable with the spectra of NIST library.

Chlorpyrifos is a crystalline organophosphate insecticide used on grain, cotton, field, fruit, nut and vegetable crops, and well as on lawns and ornamental plants. 
Table 1. Details of insecticides formulations selected for the study.

\begin{tabular}{|c|c|c|c|c|c|}
\hline $\begin{array}{l}\text { Class I } \\
\text { Group }\end{array}$ & $\begin{array}{l}\text { Active in- } \\
\text { gredients }\end{array}$ & $\begin{array}{l}\text { Formula- } \\
\text { tions }\end{array}$ & $\begin{array}{l}\text { Molecular } \\
\text { formula }\end{array}$ & IUPAC name & Purpose \\
\hline Abamectin & Emamectin & $5 \%$ SG & $\mathrm{C}_{56} \mathrm{H}_{81} \mathrm{NO}_{1}$ & $\begin{array}{l}\text { 4"-deoxy-4"-methylamino } \\
\text { derivative }\end{array}$ & $\begin{array}{l}\text { Treatment of heartworm, hook- } \\
\text { worm, threadworm, and whipworm }\end{array}$ \\
\hline \multirow[t]{3}{*}{ Ketoenols } & $\begin{array}{l}\text { Spi- } \\
\text { romesifen }\end{array}$ & $\begin{array}{l}22.9 \% \\
\mathrm{SC}\end{array}$ & $\mathrm{C}_{23} \mathrm{H}_{30} \mathrm{O}_{4}$ & $\begin{array}{l}\text { [2-oxo-3-(2,4,6- } \\
\text { trimethylphenyl)-1- } \\
\text { oxaspiro[4.4]non-3-en-4-yl] } \\
\text { 3,3-dimethylbutanoate }\end{array}$ & $\begin{array}{l}\text { Control red spiders mite, white fly in } \\
\text { tomato, chilli, brinjal, cotton and oth- } \\
\text { er crops. }\end{array}$ \\
\hline & Acetamiprid & $20 \%$ SP & $\mathrm{C}_{10} \mathrm{H}_{11} \mathrm{CIN}_{4}$ & $\begin{array}{l}\mathrm{N}-[(6-\text { chloropyridin-3-yl) } \\
\text { methyl]-N'-cyano-N- } \\
\text { methylethanimidamide }\end{array}$ & $\begin{array}{l}\text { Foliar-feeding pests such as Aphids, } \\
\text { Whiteflies, Leafhoppers, and Plant } \\
\text { bugs. }\end{array}$ \\
\hline & $\begin{array}{l}\text { Imidaclo- } \\
\text { prid }\end{array}$ & $\begin{array}{l}17.8 \% \\
\text { SC }\end{array}$ & $\begin{array}{l}\mathrm{C}_{9} \mathrm{H}_{10} \mathrm{CIN}_{5} \\
\mathrm{O}_{2}\end{array}$ & $\begin{array}{l}(N E)-N-[1-[(6-\text {-chloropyridin } \\
\text {-3-yl)methyl]imidazolidin-2- } \\
\text { ylidene]nitramide }\end{array}$ & $\begin{array}{l}\text { Control aphids, thrips, whiteflies, } \\
\text { scale, termites, turf and } \\
\text { soil insects and some beetles. }\end{array}$ \\
\hline \multirow[t]{2}{*}{ Neonicotinoid } & Thiacloprid & $\begin{array}{l}21.7 \% \\
\text { SC }\end{array}$ & $\begin{array}{l}\mathrm{C}_{10} \mathrm{H}_{9} \mathrm{CIN}_{4} \\
\mathrm{~S}\end{array}$ & $\begin{array}{l}\text { [3-[(6-chloropyridin-3-yl) } \\
\text { methyl]-1,3-thiazolidin-2- } \\
\text { ylidene]cyanamide }\end{array}$ & $\begin{array}{l}\text { Control of a variety of sucking and } \\
\text { chewing insects like aphids and } \\
\text { whiteflies }\end{array}$ \\
\hline & $\begin{array}{l}\text { Thiameth- } \\
\text { oxam }\end{array}$ & $30 \% \mathrm{FS}$ & $\begin{array}{l}\mathrm{C}_{8} \mathrm{H}_{10} \mathrm{CIN}_{5} \\
\mathrm{O}_{3} \mathrm{~S}\end{array}$ & $\begin{array}{l}\text { (NE)-N-[3-[(2-chloro-1,3- } \\
\text { thiazol-5-yl)methyl]-5- } \\
\text { methyl-1,3,5-oxadiazinan- } \\
\text { 4-ylidene]nitramide }\end{array}$ & $\begin{array}{l}\text { Controls Stem borer, gall midge, leaf } \\
\text { folder, brown plant hopper, Thrips in } \\
\text { rice and also sucking pest in cotton }\end{array}$ \\
\hline $\begin{array}{l}\text { Organochlo- } \\
\text { rine }\end{array}$ & Dicofol & $18.5 \% \mathrm{EC}$ & $\mathrm{C}_{14} \mathrm{H}_{9} \mathrm{Cl}_{5} \mathrm{O}$ & $\begin{array}{l}\text { 2,2,2-trichloro-1,1-bis }(4- \\
\text { chlorophenyl)ethanol }\end{array}$ & $\begin{array}{l}\text { Used against red spider mite in cu- } \\
\text { cumbers, ornamentals, and other } \\
\text { fruits and vegetables. }\end{array}$ \\
\hline \multirow[t]{5}{*}{$\begin{array}{l}\text { Organofluo- } \\
\text { rine }\end{array}$} & $\begin{array}{l}\text { Flubendia- } \\
\text { mide }\end{array}$ & $\begin{array}{l}39.35 \% \\
\text { SC }\end{array}$ & $\begin{array}{l}\mathrm{C}_{23} \mathrm{H}_{22} \mathrm{~F}_{7} \mathrm{IN} \\
{ }_{2} \mathrm{O}_{4} \mathrm{~S}\end{array}$ & $\begin{array}{l}1-N-[4-(1,1,1,2,3,3,3- \\
\text { heptafluoropropan-2-yl)-2- } \\
\text { methylphenyl]-3-iodo-2- } N \text { - } \\
\text { (2-methyl-1- } \\
\text { methylsulfonylpropan-2-yl) } \\
\text { benzene-1,2- } \\
\text { dicarboxamide }\end{array}$ & $\begin{array}{l}\text { Controls lepidopteron pests in rice, } \\
\text { cotton, corn, grapes, other fruits and } \\
\text { vegetables }\end{array}$ \\
\hline & Acephate & $75 \% \mathrm{SP}$ & $\begin{array}{l}\mathrm{C}_{4} \mathrm{H}_{10} \mathrm{NO}_{3} \\
\text { PS }\end{array}$ & $\begin{array}{l}N \text {-[methoxy } \\
\text { (methylsulfanyl) } \\
\text { phosphoryl]acetamide }\end{array}$ & $\begin{array}{l}\text { Used on food crops, citrus trees, as } \\
\text { a seed treatment also kills cock- } \\
\text { roaches, crickets, firebrats earwigs, } \\
\text { pillbugs, sowbugs, pantry pests, and } \\
\text { wasps. }\end{array}$ \\
\hline & $\begin{array}{l}\text { Chlorpyri- } \\
\text { fos }\end{array}$ & $20 \%$ EC & $\begin{array}{l}\mathrm{C}_{7} \mathrm{H}_{7} \mathrm{Cl}_{3} \mathrm{NO} \\
{ }_{3} \mathrm{PS}\end{array}$ & $\begin{array}{l}\text { dimethoxy-sulfanylidene- } \\
\text { (3,5,6-trichloropyridin-2-yl) } \\
\text { oxy-lambda5-phosphane } \\
\text { 2- }\end{array}$ & $\begin{array}{l}\text { Control cutworms, corn rootworms, } \\
\text { cockroaches, grubs, flea beetles, } \\
\text { flies, termites, fire ants, and lice }\end{array}$ \\
\hline & Dimethoate & $30 \%$ EC & $\begin{array}{l}\mathrm{C}_{5} \mathrm{H}_{12} \mathrm{NO}_{3} \\
\mathrm{PS}_{2}\end{array}$ & $\begin{array}{l}\text { dimethoxyphosphinothi- } \\
\text { oylsulfanyl- } N \text { - } \\
\text { methylacetamide }\end{array}$ & $\begin{array}{l}\text { Used against sucking insects like } \\
\text { aphids, leafhoppers, and thrip }\end{array}$ \\
\hline & Ethion & $50 \%$ EC & $\begin{array}{l}\mathrm{C}_{9} \mathrm{H}_{22} \mathrm{O}_{4} \mathrm{P}_{2} \\
\mathrm{~S}_{4}\end{array}$ & $\begin{array}{l}\text { diethoxyphosphinothi- } \\
\text { oylsulfanylmethylsulfanyl- } \\
\text { diethoxy-sulfanylidene- } \lambda 5- \\
\text { phosphane }\end{array}$ & $\begin{array}{l}\text { Used to kill aphids, mites, scales, } \\
\text { thrips, leafhoppers, maggots and } \\
\text { foliar feeding larvae. }\end{array}$ \\
\hline \multirow{4}{*}{$\begin{array}{l}\text { Organophos- } \\
\text { phate }\end{array}$} & $\begin{array}{l}\text { Monocroto- } \\
\text { phos }\end{array}$ & $36 \% \mathrm{SL}$ & $\begin{array}{l}\mathrm{C}_{7} \mathrm{H}_{14} \mathrm{NO}_{5} \\
P\end{array}$ & $\begin{array}{l}\text { dimethyl [(E)-4- } \\
\text { (methylamino)-4-oxobut-2- } \\
\text { en-2-yl] phosphate }\end{array}$ & $\begin{array}{l}\text { Control sucking, chewing and boring } \\
\text { insects and spider mites on cotton, } \\
\text { sugarcane, peanuts, ornamentals, } \\
\text { and tobacco }\end{array}$ \\
\hline & Profenofos & $50 \%$ EC & $\begin{array}{l}\mathrm{C}_{11} \mathrm{H}_{15} \mathrm{Br}- \\
\mathrm{ClO}_{3} \mathrm{P}\end{array}$ & $\begin{array}{l}\text { 4-bromo-2-chloro-1- } \\
\text { [ethoxy(propylsulfanyl) } \\
\text { phosphoryl]oxybenzene }\end{array}$ & $\begin{array}{l}\text { Control over all sucking pests and } \\
\text { foliar feeding larvae and control of } \\
\text { mites on a variety of crops }\end{array}$ \\
\hline & Triazophos & $40 \%$ EC & $\begin{array}{l}\mathrm{C}_{12} \mathrm{H}_{16} \mathrm{~N}_{3} \mathrm{O} \\
{ }_{3} \mathrm{PS}\end{array}$ & $\begin{array}{l}\text { diethoxy-[(1-phenyl-1,2,4- } \\
\text { triazol-3-yl)oxy]- } \\
\text { sulfanylidene-lambda5- } \\
\text { phosphane }\end{array}$ & $\begin{array}{l}\text { Controls Aphids, thrips, midges, bee- } \\
\text { tles, larvae, cutworms, and other soil } \\
\text { insects in cereals, sugarbeets, sug- } \\
\text { arcanes, maize, soybeans, coffee, } \\
\text { and grasslands. }\end{array}$ \\
\hline & Quinalphos & $25 \%$ EC & $\begin{array}{l}\mathrm{C}_{12} \mathrm{H}_{15} \mathrm{~N}_{2} \mathrm{O} \\
{ }_{3} \mathrm{PS}\end{array}$ & $\begin{array}{l}\text { diethoxy-quinoxalin-2- } \\
\text { yloxy-sulfanylidene- } \\
\text { lambda5-phosphane }\end{array}$ & $\begin{array}{l}\text { Toxic against bollworms on cotton } \\
\text { and stem borer, green leaf hopper, } \\
\text { hispa on rice }\end{array}$ \\
\hline
\end{tabular}


Table 1. Contd.....

\begin{tabular}{|c|c|c|c|c|c|}
\hline $\begin{array}{l}\text { Phenylpyra- } \\
\text { zole }\end{array}$ & Fipronil & $5 \% \mathrm{SC}$ & $\begin{array}{l}\mathrm{C}_{12} \mathrm{H}_{4} \mathrm{Cl}_{2} \mathrm{~F}_{6} \\
\mathrm{~N}_{4} \mathrm{OS}\end{array}$ & $\begin{array}{l}\text { 5-amino-1-[2,6-dichloro-4- } \\
\text { (trifluoromethyl)phenyl]-4- } \\
\text { (trifluoromethylsulfinyl) } \\
\text { pyrazole-3-carbonitrile }\end{array}$ & $\begin{array}{l}\text { Used to control ants, beetles, cock- } \\
\text { roaches, fleas, ticks, termites, mole } \\
\text { crickets, thrips, rootworms, weevils, } \\
\text { and other insects }\end{array}$ \\
\hline \multirow[b]{2}{*}{ Pyrethroids } & $\begin{array}{l}\text { Cyperme- } \\
\text { thrin }\end{array}$ & $10 \%$ EC & $\begin{array}{l}\mathrm{C}_{22} \mathrm{H}_{19} \mathrm{Cl}_{2} \mathrm{~N} \\
\mathrm{O}_{3}\end{array}$ & $\begin{array}{l}\text { [cyano-(3-phenoxyphenyl) } \\
\text { methyl] 3-(2,2- } \\
\text { dichloroethenyl)-2,2- } \\
\text { dimethylcyclopropane-1- } \\
\text { carboxylate }\end{array}$ & $\begin{array}{l}\text { To kill insects on cotton and lettuce, } \\
\text { and to kill cockroaches, fleas, and } \\
\text { termites in houses and other build- } \\
\text { ings. }\end{array}$ \\
\hline & $\begin{array}{l}\text { Lambda } \\
\text { Cyhalothrin }\end{array}$ & $5 \%$ EC & $\begin{array}{l}\mathrm{C}_{23} \mathrm{H}_{19} \mathrm{ClF}_{3} \\
\mathrm{NO}_{3}\end{array}$ & $\begin{array}{l}\text { [(R)-cyano-(3- } \\
\text { phenoxyphenyl)methyl] } \\
\text { (1S,3S)-3-[(Z)-2-chloro- } \\
\text { 3,3,3-trifluoroprop-1-enyl]- } \\
\text { 2,2-dimethylcyclopropane- } \\
\text { 1-carboxylate }\end{array}$ & $\begin{array}{l}\text { To control aphids, Colorado beetles } \\
\text { and butterfly larvae }\end{array}$ \\
\hline Quinazoline & Fenzaquin & $10 \%$ EC & $\mathrm{C}_{20} \mathrm{H}_{22} \mathrm{~N}_{2} \mathrm{O}$ & $\begin{array}{l}\text { 4-[2-(4-tert-butylphenyl) } \\
\text { ethoxy]quinazoline }\end{array}$ & $\begin{array}{l}\text { Used against a broad spectrum of } \\
\text { mites in grapes, pome fruit, citrus, } \\
\text { peaches, cucurbits, tomatoes, cotton } \\
\text { and ornamentals }\end{array}$ \\
\hline Thiourea & $\begin{array}{l}\text { Diafenthi- } \\
\text { uron }\end{array}$ & $50 \%$ WP & $\begin{array}{l}\mathrm{C}_{23} \mathrm{H}_{32} \mathrm{~N}_{2} \mathrm{O} \\
\mathrm{S}\end{array}$ & $\begin{array}{l}\text { 1-tert-butyl-3-[4-phenoxy- } \\
\text { 2,6-di(propan-2-yl)phenyl] } \\
\text { thiourea }\end{array}$ & $\begin{array}{l}\text { It is toxic to cardamom borer, Cono- } \\
\text { gethes punctiferalis, Guenee, Indian } \\
\text { bees. }\end{array}$ \\
\hline
\end{tabular}

Table 2: Insecticide determination using FTIR Spectrometry in ATR and KBr method.

\begin{tabular}{|c|c|c|c|c|c|}
\hline Insecticides & $\begin{array}{l}\text { Measurement } \\
\text { mode }\end{array}$ & $\begin{array}{l}\text { Wave number } \\
\text { range }\left(\mathrm{cm}^{-1}\right)\end{array}$ & Baseline $\left(\mathrm{cm}^{-1}\right)$ & $\begin{array}{l}\text { Sample through- } \\
\text { put }\left(\mathrm{hr}^{-1}\right)\end{array}$ & $\begin{array}{l}\text { Waste genera- } \\
\text { tion }\end{array}$ \\
\hline Acephate & $\mathrm{KBr}$ disks & $1700-1025$ & $1750-500$ & $5-6$ & $10-20 \mathrm{mg}$ \\
\hline Acetamiprid & $\mathrm{KBr}$ disks & $1532-600$ & $2200-500$ & $5-6$ & $10-20 \mathrm{mg}$ \\
\hline Chlorpyrifos & ATR & $1022-580$ & $1500-600$ & $8-9$ & $0.5-1 \mathrm{ml}$ \\
\hline Cypermethrin & ATR & $2200-1500$ & 1500 & $8-9$ & $0.5-1 \mathrm{ml}$ \\
\hline Diafenthiuron & $\mathrm{KBr}$ disks & $1740-1160$ & $1200-500$ & $5-6$ & $10-20 \mathrm{mg}$ \\
\hline Dicofol & ATR & $1480-600$ & $3000-600$ & $8-9$ & $0.5-1 \mathrm{ml}$ \\
\hline Dimethoate & ATR & $1070-580$ & $1400-600$ & $8-9$ & $0.5-1 \mathrm{ml}$ \\
\hline Emamectin & $\mathrm{KBr}$ disks & $2800-1250$ & $2500-3500$ & $5-6$ & $10-20 \mathrm{mg}$ \\
\hline Ethion & ATR & $2865-1375$ & $1500-1000$ & $8-9$ & $0.5-1 \mathrm{ml}$ \\
\hline Fenzaquin & ATR & $3080-1430$ & $1600-1300$ & $8-9$ & $0.5-1 \mathrm{ml}$ \\
\hline Fipronil & ATR & $1625-600$ & $1800-600$ & $8-9$ & $0.5-1 \mathrm{ml}$ \\
\hline Flubendiamide & ATR & $2700-910$ & $3000-800$ & $8-9$ & $0.5-1 \mathrm{ml}$ \\
\hline Imidacloprid & ATR & $1680-1550$ & $1600-600$ & $8-9$ & $0.5-1 \mathrm{ml}$ \\
\hline $\begin{array}{l}\text { Lambda Cyhalo- } \\
\text { thrin }\end{array}$ & ATR & $1300-1110$ & $1500-900$ & $8-9$ & $0.5-1 \mathrm{ml}$ \\
\hline Monocrotophos & ATR & $1680-1200$ & $1700-1100$ & $8-9$ & $0.5-1 \mathrm{ml}$ \\
\hline Profenofos & ATR & $1485-600$ & $1400-600$ & $8-9$ & $0.5-1 \mathrm{ml}$ \\
\hline Quinalphos & ATR & $1090-810$ & $1100-800$ & $8-9$ & $0.5-1 \mathrm{ml}$ \\
\hline Spiromesifen & ATR & $1680-1020$ & $1200-100$ & $8-9$ & $0.5-1 \mathrm{ml}$ \\
\hline Thiacloprid & ATR & $1650-1550$ & $1600-1400$ & $8-9$ & $0.5-1 \mathrm{ml}$ \\
\hline Thiamethoxam & ATR & $1635-1310$ & $1500-900$ & $8-9$ & $0.5-1 \mathrm{ml}$ \\
\hline Triazophos & ATR & $1525-1020$ & $1500-600$ & $8-9$ & $0.5-1 \mathrm{ml}$ \\
\hline
\end{tabular}


The halogen group showed a strong stretching of $\mathrm{C}-\mathrm{Cl}$ bond at $830-600 \mathrm{~cm}^{-1}$ and $\mathrm{P}=\mathrm{S}$ bond showed variable strong stretching at $800-580 \mathrm{~cm}^{-1}$. In most instances, when used alone, strong absorption at the cited regions was considered to be related to the stretching vibration of only a C-O- link. Another absorption feature of the spectrum that should be taken into consideration was C-O- absorption band, which was indicative of an ether group. For example, the absence of characteristic absorption features of those functional groups that contained the C-O- link (alcohol groups, ester groups, etc.) increases the probability of the C-Oabsorption as indicative of an ether group. On equating chlorpyriphos 20\%EC (Fig.10b)spectra with the NIST spectra and KnowitAll (Fig.10f) software, peak at $1022.57 \mathrm{~cm}^{-1}$ shows $100 \%$ intensity and at $1410.67 \mathrm{~cm}$ ${ }^{-1}$ shows $69.13 \%$. The absorption bands of $\mathrm{C}-\mathrm{N}$ stretching, C-Cl stretching and P-S stretching for chlorpyriphos was also reported by Armenta et al. (2005a).

Dimethoate is a broadly utilized organophosphate bug spray and acaricide. Dimethoate 30\% EC IR (Fig.10c) spectrum analyzed with bio-rad software (Fig.10g) showed that it had the strong stretching of $\mathrm{P}=\mathrm{S}$ bond in the range of 800 to $580 \mathrm{~cm}^{-1}$ and the intensity was of $97.24 \%$ at $783.92 \mathrm{~cm}^{-1} .100 \%$ intensity is available at $1070.30 \mathrm{~cm}^{-1}$ band, which is corroborated with NIST library spectrum. Medium bending and stretching of

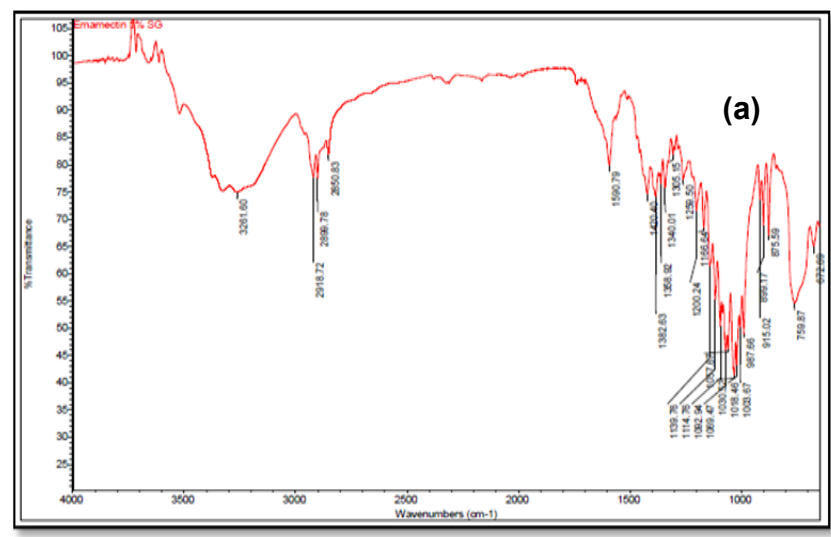

$\mathrm{NH}$ and $\mathrm{C}-\mathrm{N}$ bonds were observed at 1440-1490 and $1310-1350 \mathrm{~cm}^{-1}$.

Ethion is used on a wide variety of food, fiber and ornamental crops, including greenhouse crops, lawns and turf. Ethion $50 \%$ EC (Fig.10d) contained $\mathrm{P}=\mathrm{S}$ group and has a stretching at $800-580 \mathrm{~cm}^{-1}$ and string symmetric $\mathrm{CH}$ group at 2863-2843 $\mathrm{cm}^{-1}$, medium symmetric $\mathrm{CH}$ group at $1380-1375 \mathrm{~cm}^{-1}$. The intensity ranges from $48.87 \%$ to $82.95 \%$ in the area of $\mathrm{P}=\mathrm{S}$ group as observed by ProcessItIR (Fig.10h) and Spectrabase. Similar vibration features of ethion at 720 and $1718 \mathrm{~cm}^{-1}$ due to $\mathrm{P}=\mathrm{S}$ vibrations and $\mathrm{S}-\mathrm{P}=\mathrm{S}$ stretching, respectively was reported by Yang et al. (2019).

Monocrotophos 36\% SL spectra showed (Fig. 11a) signature bands (Fig. 11e) of $\mathrm{N}-\mathrm{H}$ stretching vibration near $3270 \mathrm{~cm}^{-1}$, very strong $\mathrm{C}=\mathrm{O}$ stretching vibration at $1680-1630 \mathrm{~cm}^{-1}$, strong intensity of $\mathrm{NH}$ deformation and C-N stretching at $1570-1515 \mathrm{~cm}^{-1}$ and mixed C-N stretching and $\mathrm{N}-\mathrm{H}$ bending at $1310-1200 \mathrm{~cm}^{-1}$. Variable stretching of P-O-R bond was observed at 1050$970 \mathrm{~cm}^{-1}$.

Profenofos (Fig. 11b) derived from a 4-bromo-2chlorophenol showed antisymmetric stretching of $\mathrm{CH}$ bond at 2936-2916 $\mathrm{cm}^{-1}$, symmetric stretching of $\mathrm{CH}$ bond at 2863-2843 $\mathrm{cm}^{-1}$, and deformation of $\mathrm{CH}$ bond at $1485-1445 \mathrm{~cm}^{-1}$ in aromatic $1,2,3$ trisubstituted ring. Strong stretching of halogen bonds of $\mathrm{C}-\mathrm{Br}$ and $\mathrm{C}-\mathrm{Cl}$ at

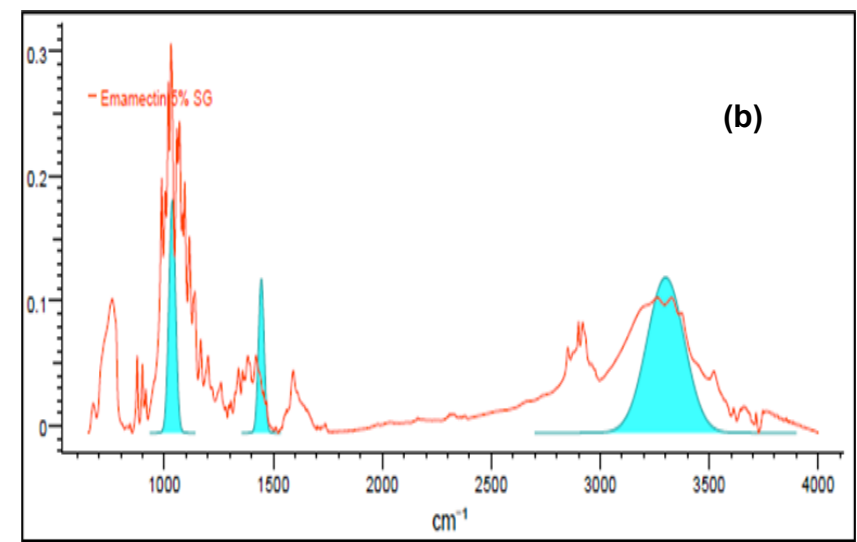

Fig. 2. IR spectra of Emamectin (a- Original spectrum; b). Processed spectrum by KnowitAll).
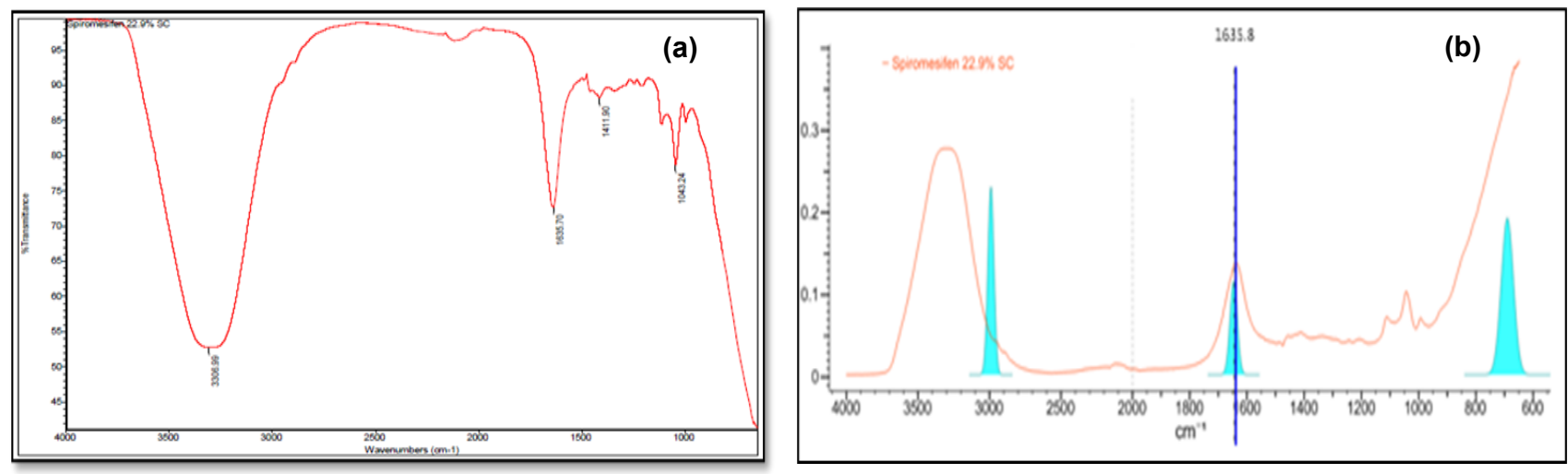

Fig. 3. IR spectra of Spiromesifen (a). Original spectrum; b). Processed spectrum by KnowitAll). 
500-550 and 830-600 $\mathrm{cm}^{-1}$ respectively was also seen in spectra(Fig.11f). It showed $100 \%$ intensity at $1472.87 \mathrm{~cm}^{-1}$ for $\mathrm{CH}$ bond of alkanes.

Quinalphos (derives from a quinoxalin-2-ol)spectrum (Fig.11C)peak bands at 800-580, 810-870, 990-1090 and $2340-2790 \mathrm{~cm}^{-1}$ showed the presence of $\mathrm{P}=\mathrm{S}$ group stretching, $\mathrm{S}-\mathrm{O}$ bond stretching, $\mathrm{S}=\mathrm{O}$ bond and $\mathrm{O}-\mathrm{H}$ bond stretching, respectively (Fig.11g). The 100\% peak intensity was obtained at $1023.05 \mathrm{~cm}^{-1}$.

Triazophos is an acaricide,derived from a 1-phenyl-1H1,2,4-triazol-3-ol. Triazophos 40\% EC spectrum (Fig.11d)was compared with the spectrabase and biorad software (Fig. $11 \mathrm{~h}$ ). It showed variable stretching of $\mathrm{P}=\mathrm{S}$ bond at $580-800 \mathrm{~cm}^{-1}$, strong bending of an aro-

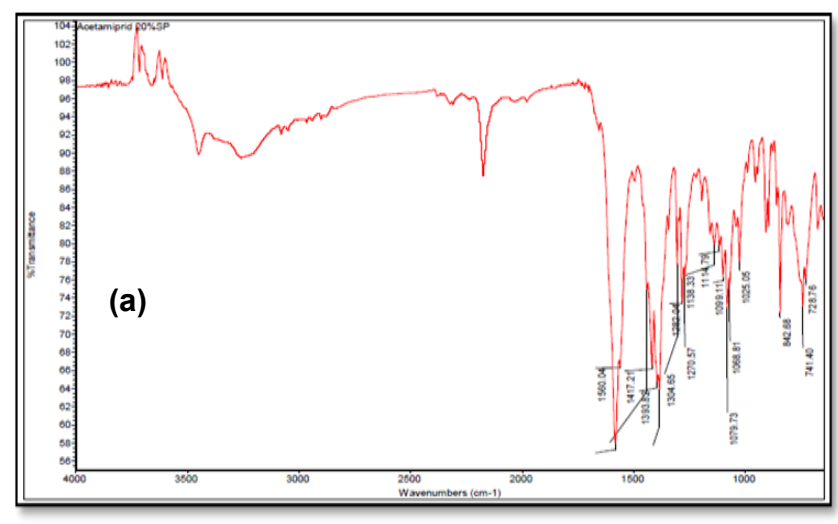

matic ring at $690-710 \mathrm{~cm}^{-1}$ and strong antisymmetric C$\mathrm{H}$ bond of alkanes at $2952-2972 \mathrm{~cm}^{-1}$. It showed $100 \%$ intensity at $1019.38 \mathrm{~cm}^{-1}, 94.93 \%$ at $1524.73 \mathrm{~cm}^{-1}$ and $86.61 \%$ at $1329.92 \mathrm{~cm}^{-1}$.

\section{Phenylpyrazoles}

Fipronil is utilized to control ants, beetles, cockroaches, bugs, ticks, termites, mole crickets, thrips, rootworms, weevils, and different bugs. Fipronil 5\% SC (Fig.12a) contains variable to medium stretching of aromatics ring group in the range of $1430-1625 \mathrm{~cm}^{-1}$ and halogen groups of $\mathrm{C}-\mathrm{Cl}$ and $\mathrm{C}-\mathrm{F}$ groups in the range of 830$600 \mathrm{~cm}^{-1}$ and $1300-900 \mathrm{~cm}^{-1}$ of strong and variable stretching. The peak intensity at $711.12 \mathrm{~cm}^{-1}$ showed

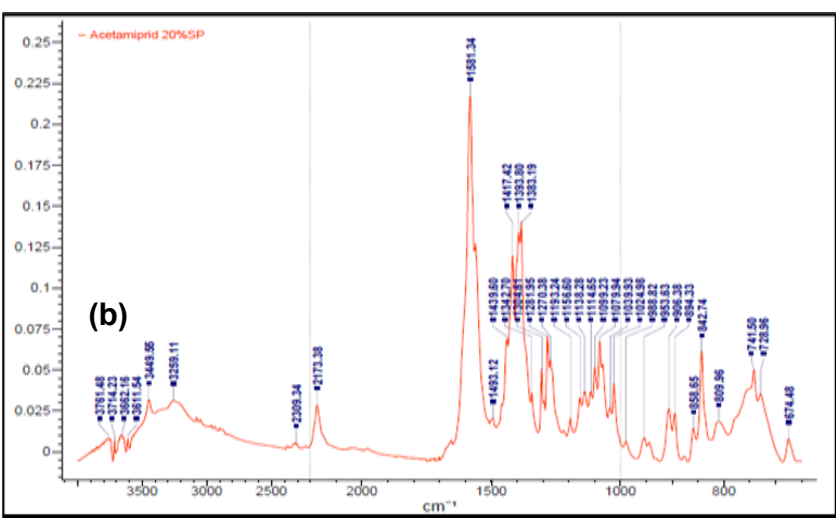

Fig. 4. IR spectra of Acetamiprid (a). Original spectrum; b). Processed spectrum by KnowitAll).
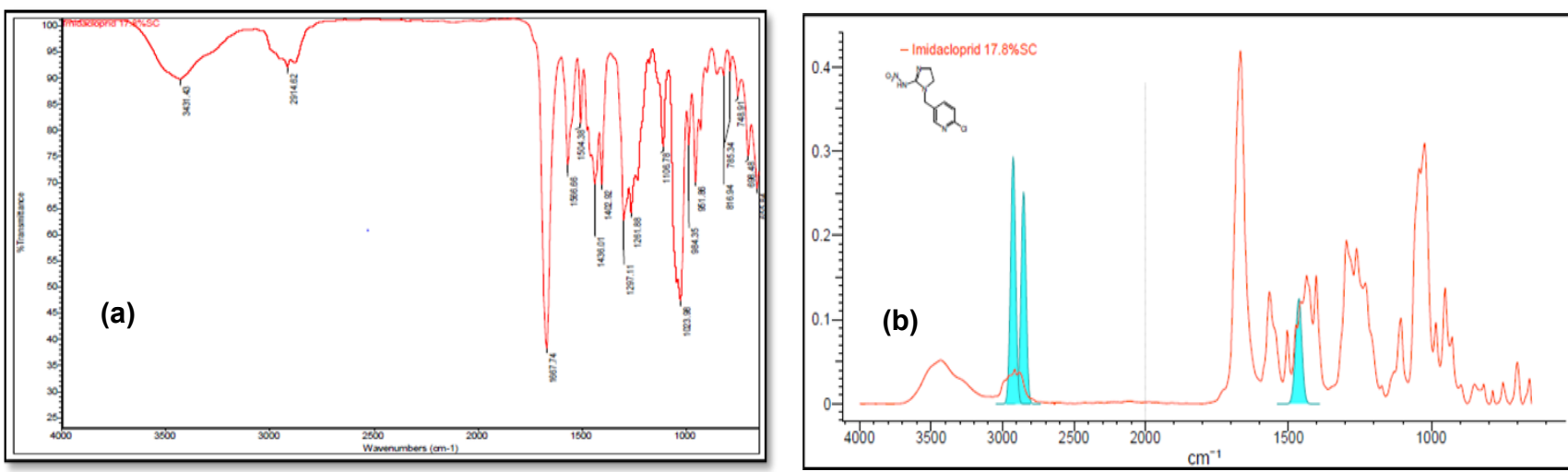

Fig. 5. IR spectra of Imdiacloprid (a). Original spectrum; b). Processed spectrum by KnowitAll).
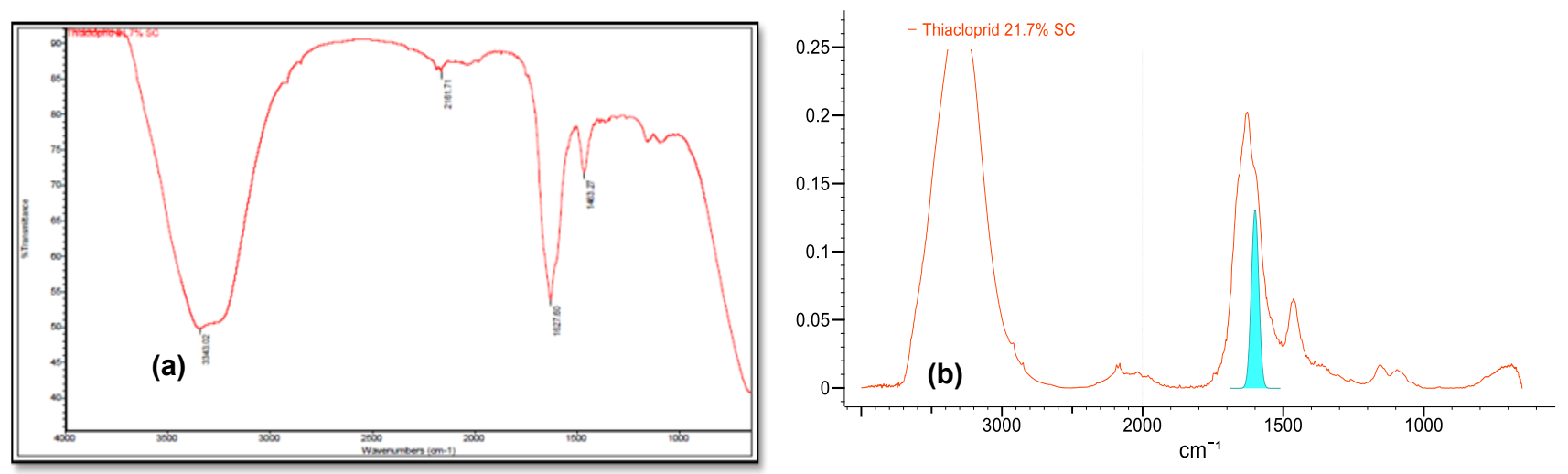

Fig. 6. IR spectra of Thiocloprid (a). Original spectrum; b). Processed spectrum by KnowitAll). 

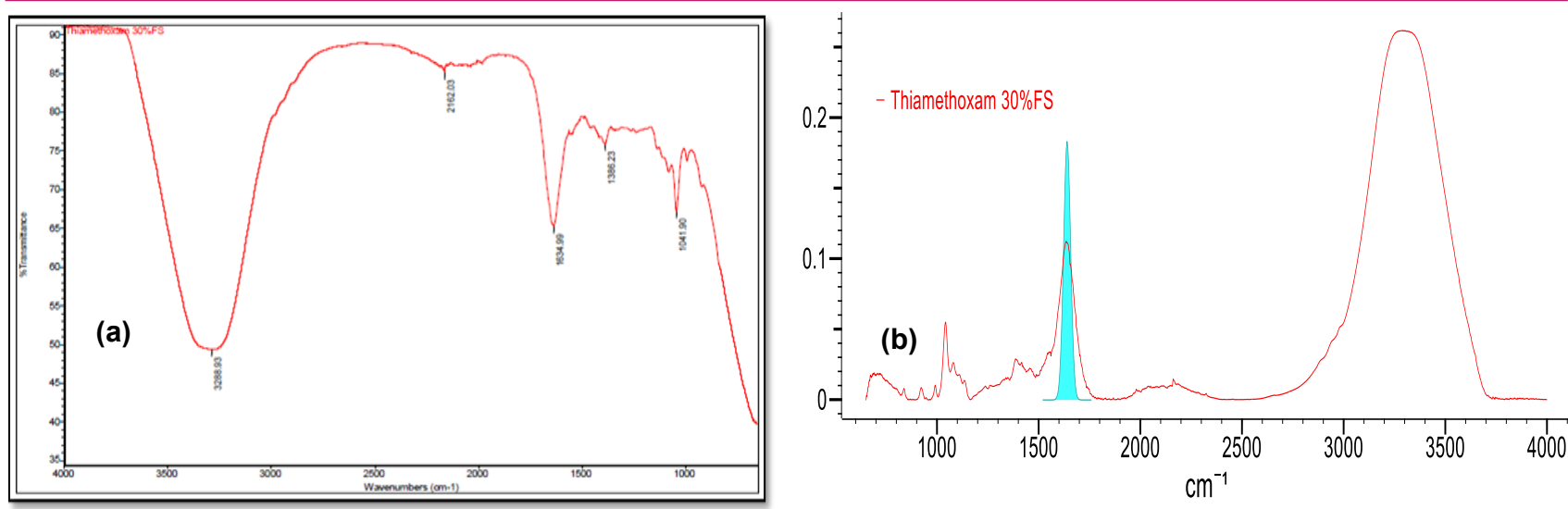

Fig. 7. IR spectra of Thiomethoxam (a). Original spectrum; b). Processed spectrum by KnowitAll).
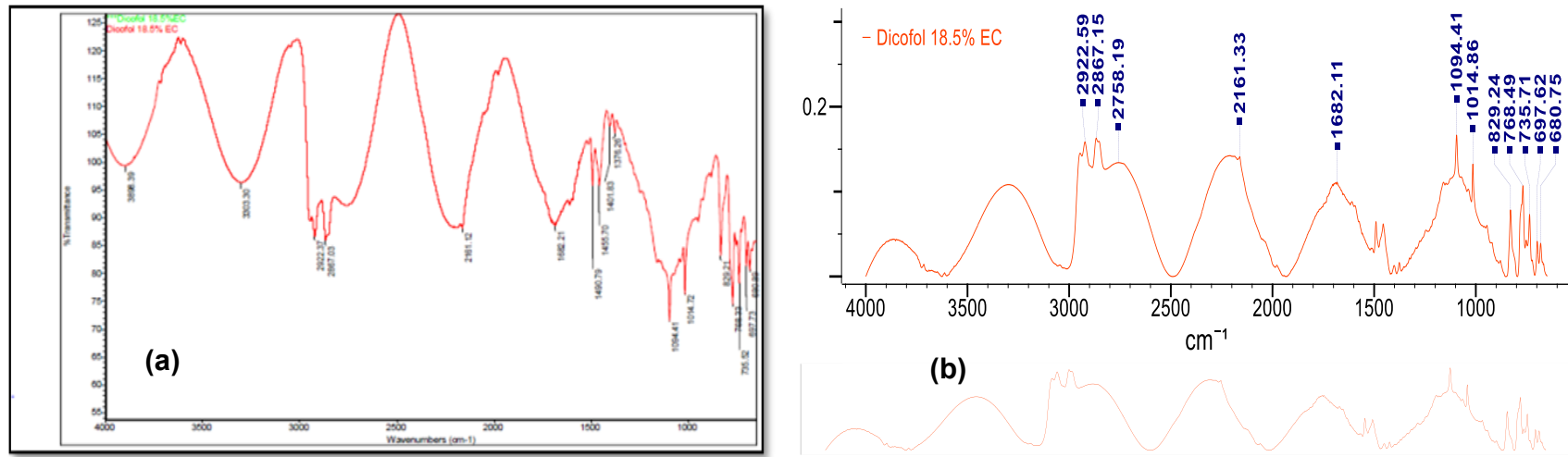

(b)

Fig. 8. IR spectra of Dicofol (a). Original spectrum; b). Processed spectrum by KnowitAll).
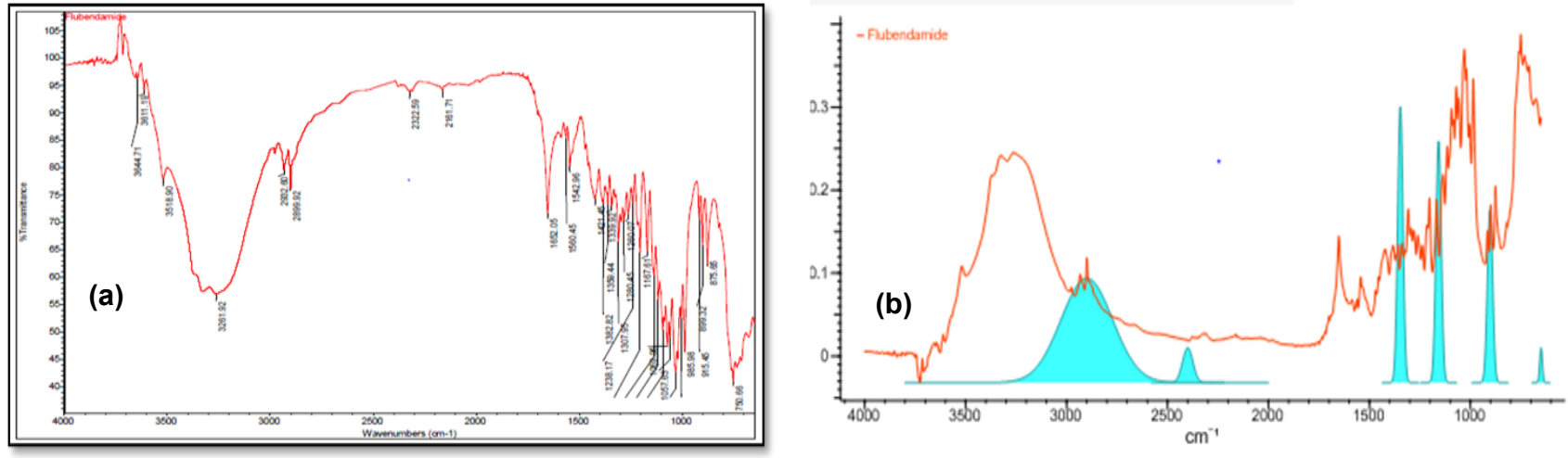

Fig. 9. IR spectra of Flubendamide (a). Original spectrum; b). Processed spectrum by KnowitAll).

$100 \%$ by $\mathrm{C}-\mathrm{Cl}$ group and $1070.30 \mathrm{~cm}^{-1}$ has an intensity of $23.06 \%$. The commercial spectra were compared with the spectrabase and bio-rad software (Fig.12b) also. Two major absorption peaks at 1633 and $1319 \mathrm{~cm}$ ${ }^{-1}$ for fipronil was reported due to stretching vibrations of $\mathrm{C}-\mathrm{N}$ bond and deformation vibrations of $\mathrm{N}-\mathrm{H}$ bond, respectively(Qiu et al., 2013)

\section{Pyrethroids}

Cypermethrin is an engineered pyrethroid spray used to kill cockroaches, bugs, and termites in houses and different structures. Cypermethrin 10\% EC (Fig.13a) Processing bybio-rad software (Fig.13b) shows the peak area from $860-2200 \mathrm{~cm}^{-1}$ which indicates the presence of $\mathrm{C}-\mathrm{Cl}$ bond and the amine group in it. The

finger print bands of cypermethrin at 865, 1454, 1586 $\mathrm{cm}^{-1}$ due to deformation vibrations of the cyclopropane ring, $\mathrm{R}-\mathrm{CH}_{2}-\mathrm{CN}$ deformation structure and $\mathrm{C}-\mathrm{C}$ stretching of the aromatic rings as proposed by Segal-Rosenheimer and Dubowski (2007) was observed in this study. Additional important representing band of the molecule was observed at $1124 \mathrm{~cm}^{-1}$ and is related to the $\mathrm{CN}-\mathrm{O}$ stretching of the cyanate group. Segal-Rosenheimer and Dubowski (2007) reported that the absorption bands at 1742, 1587,1488, 1449 and $1076 \mathrm{~cm}^{-1}$, due to carbonyl stretching, C-C stretching in chloroalkenes, ring vibration of benzene, $\mathrm{CH}_{2}$ deformation in $\mathrm{R}-\mathrm{CH}_{2}-\mathrm{CN}$ structure and (C O)-Ostretching, respectively for pure certified standard cypermethrin. 
Asan Mohamed, B. and Janaki, P. / J. Appl. \& Nat. Sci. 13 (SI), 110 - 123 (2021)
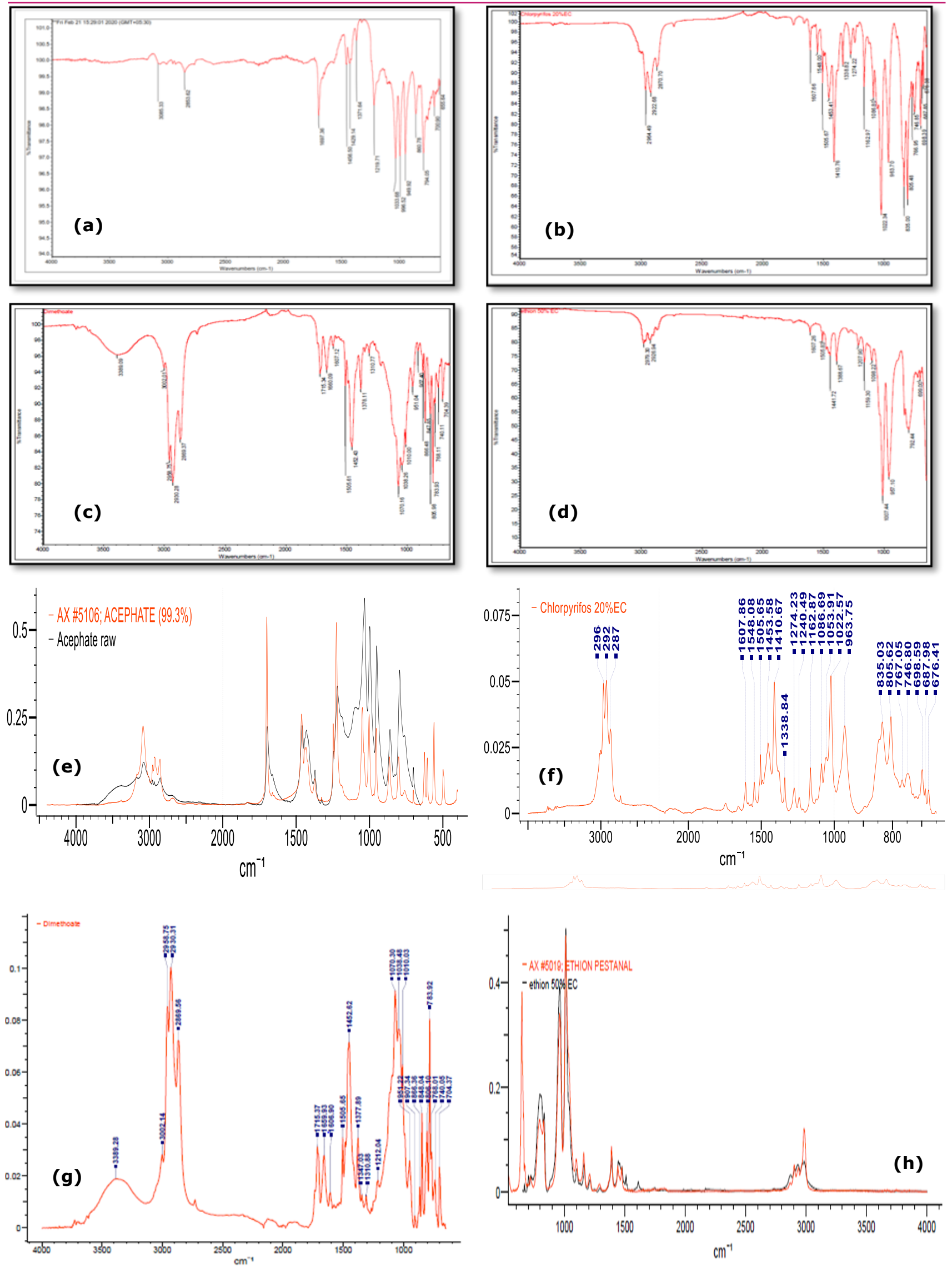

Fig. 10. IR spectra of Acephate, Chlorpyrifos, Dimethoate and Ethion.(1).Original spectrum: a, b, c, d; 2).Processed spectrum by KnowitAll: $e, f, g, h$. 
Asan Mohamed, B. and Janaki, P. / J. Appl. \& Nat. Sci. 13 (SI), 110 - 123 (2021)
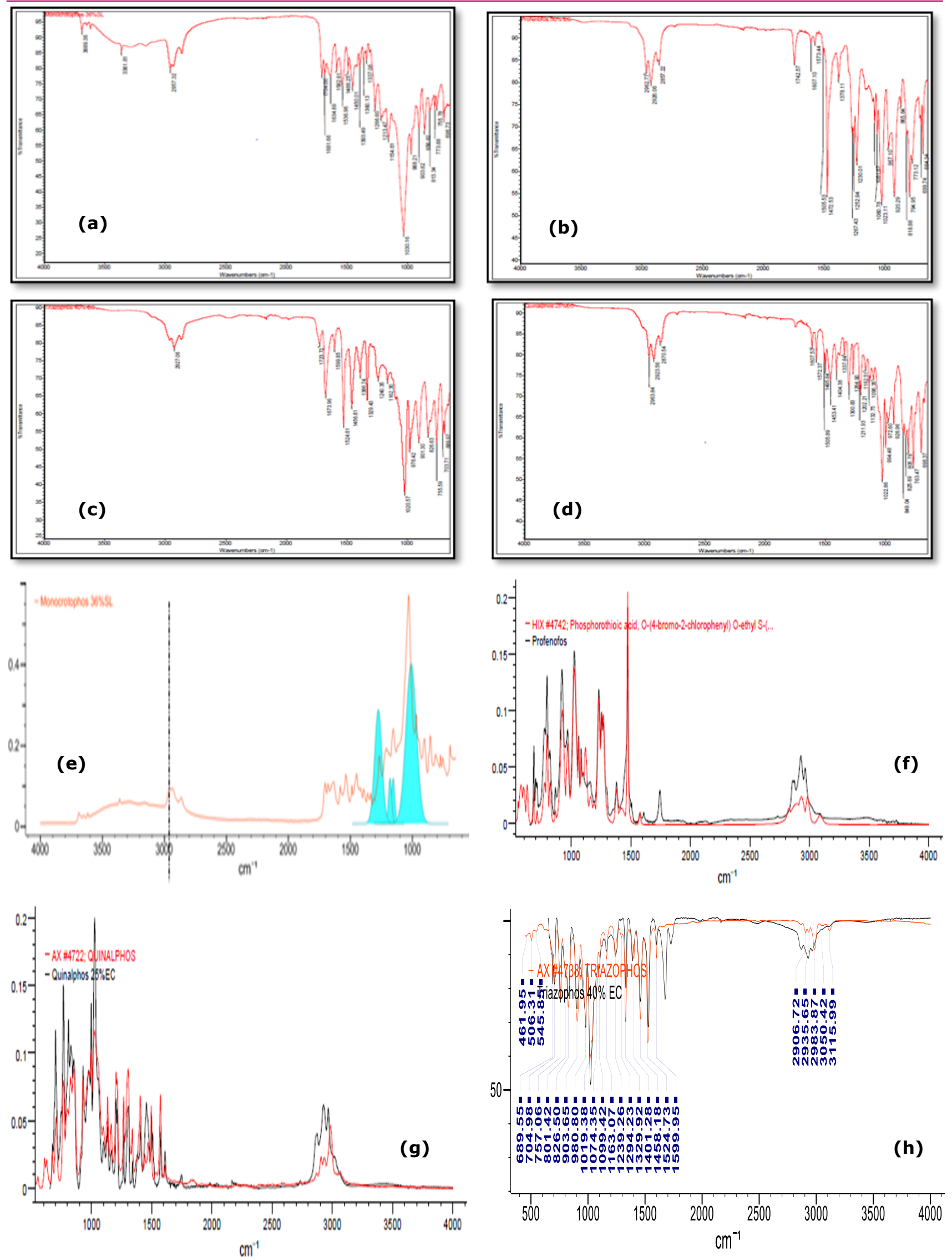

Fig. 11. IR spectra of Monocrotophos, Profenofos, QuinalphosandTriazophos.(1).Original spectrum: a, b, c, d; 2). Processed spectrum by KnowitAll: $e, f, g, h)$. 

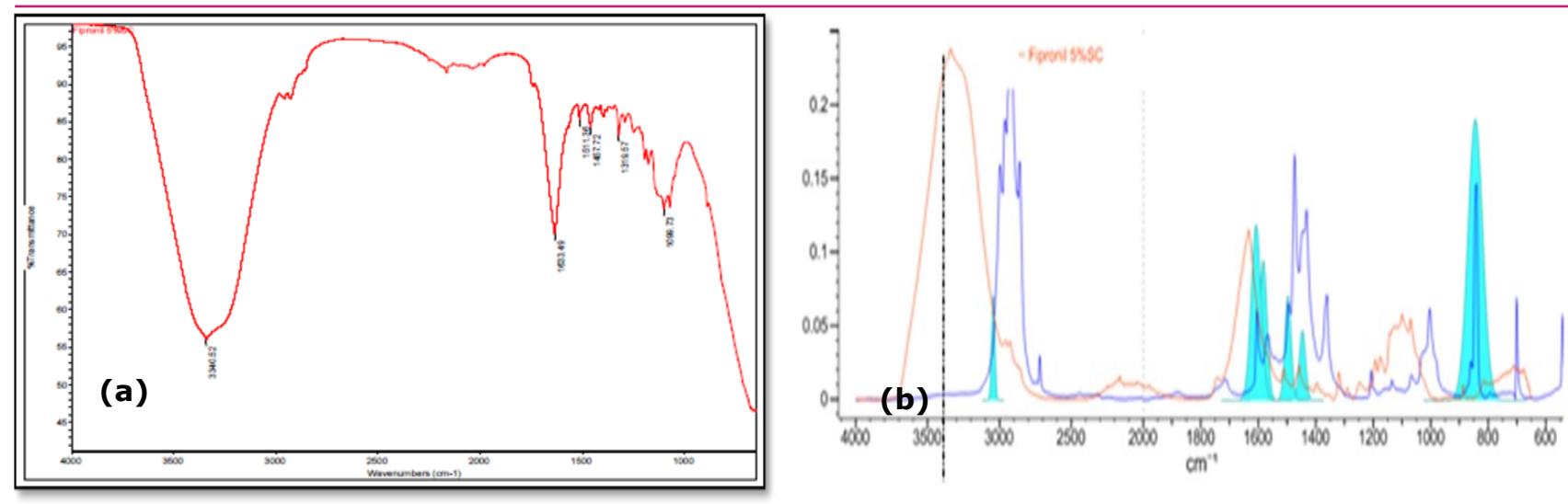

Fig. 12. IR spectra of fipronil (a). Original spectrum; b). Processed spectrum by KnowitAll).
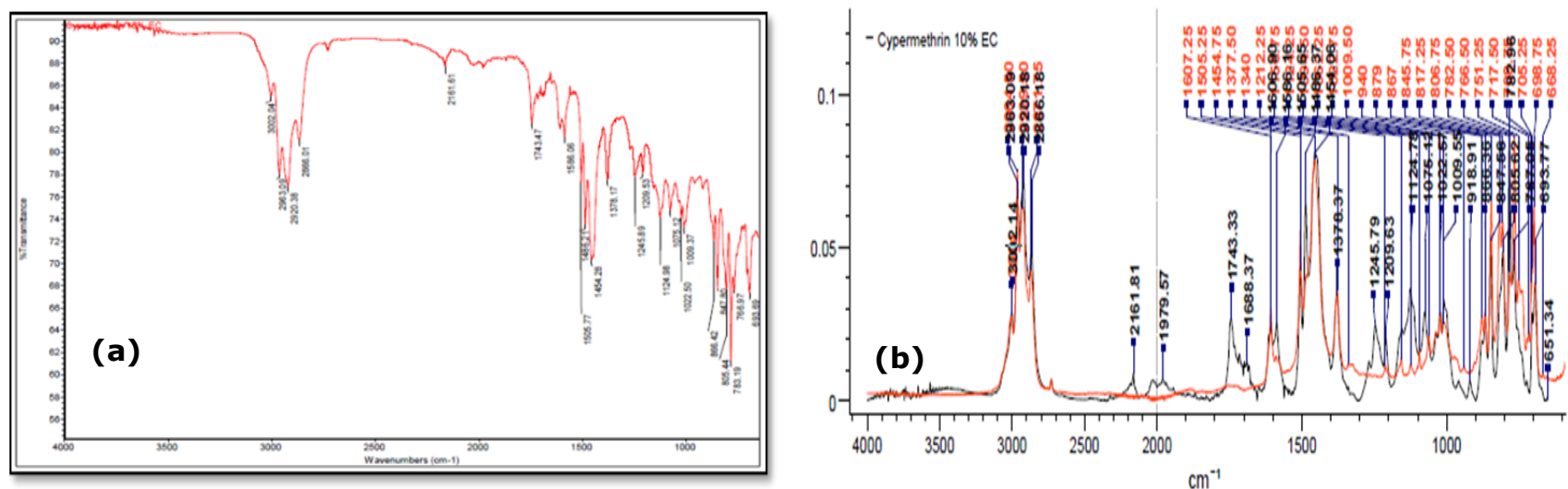

Fig. 13. IR spectra of cypermethrin(a). Original spectrum; b). Processed matching spectrum by spectrabase.
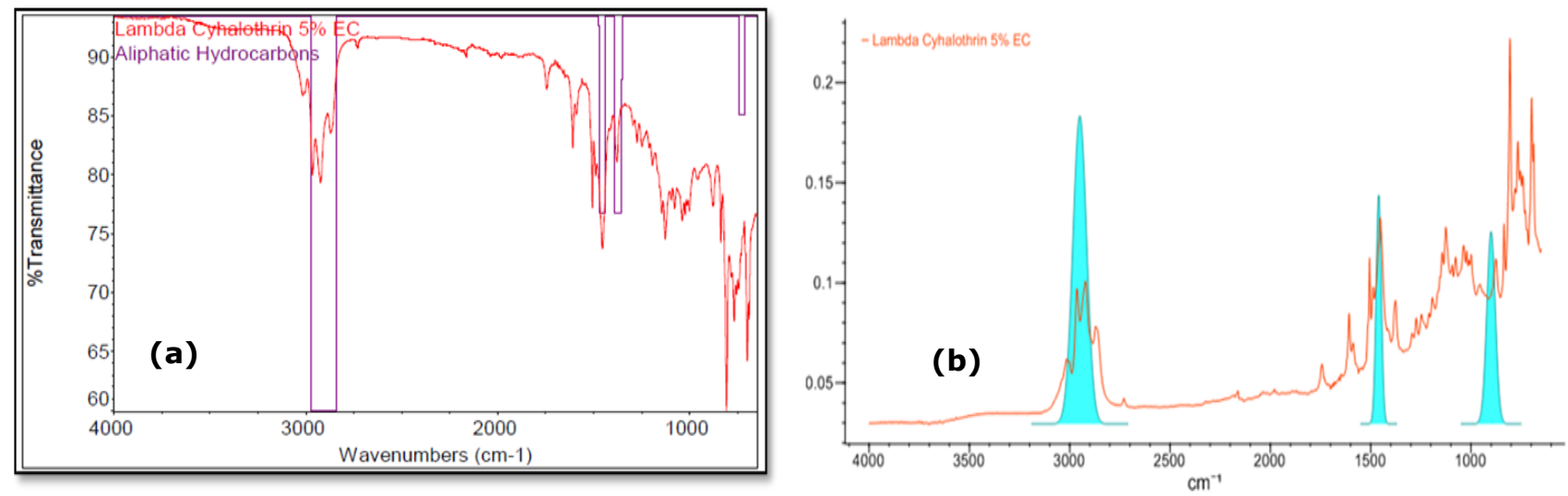

Fig. 14. IR of cyhalothrin (a). Original spectrum; b). Processed spectrum by KnowitAll).

Cyhalothrin, is a cyano-(3-phenoxyphenyl)methyl] 3(2,2-dichloroethenyl)-2,2-dimethylcyclopropane-1-

carboxylate. Commercial product spectrum (Fig.14a and $14 \mathrm{~b}$ ) showed the presence of aliphatic hydrocarbons, halogen groups, sulphur and phosphorus compounds. Symmetric stretching of $\mathrm{CH}$ bond and deformation was observed at the band of 2900-3000 and 1490-1430 $\mathrm{cm}^{-1}$ respectively. The halogen C-F bond was present at $1350-1120 \mathrm{~cm}^{-1}$ and $780-680 \mathrm{~cm}^{-}$ 1 bands. The symmetric stretching of $\mathrm{R}-\mathrm{N}=\mathrm{S}=\mathrm{O}$ at $1180-1110 \mathrm{~cm}^{-1}$ and $\mathrm{P}=\mathrm{N}$ bond stretching at 1300 $1100 \mathrm{~cm}^{-1}$ was observed as signature bands for cyhalothrin.

\section{Quinazolines}

Fenzaquin, a 4-[2-(4-tert-butylphenyl)ethoxy]quinazo line is the active ingredient of Fenzaquin $10 \%$ EC (Fig.15a). FTIR Spectrum contains aromatics o-disubstituted ring (Fig.15b) in the peak range of 1430 $1625 \mathrm{~cm}^{-1}, \mathrm{CH}$ group stretching at $3079-3010 \mathrm{~cm}^{-1}$. The peak intensity was $56.97 \%$ at $1495.53 \mathrm{~cm}^{-1}$ and $56.88 \%$ at $1454.06 \mathrm{~cm}^{-1}$ due to variable, medium stretching of aromatic string.

\section{Thioureas}

The absorbance FTIR spectra of diafenthiuron 50\% WP in the wavenumber region from 4000 to $900 \mathrm{~cm}^{-1}$ was 

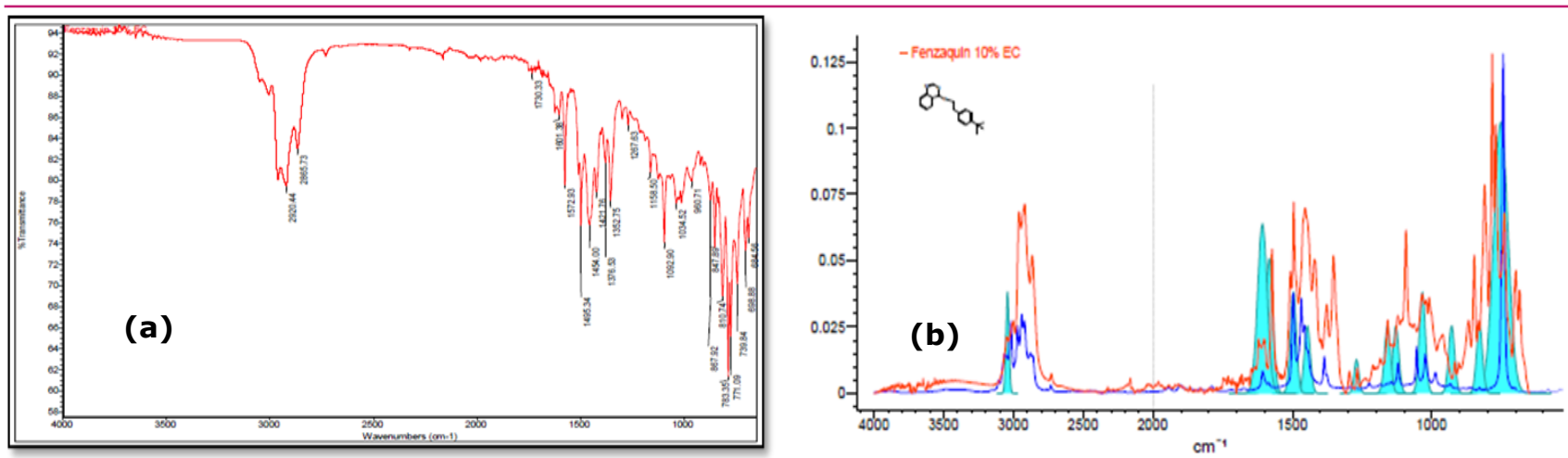

Fig. 15. IR spectra of Fenzaquin (a). Original spectrum; b). Processed spectrum by KnowitAll).
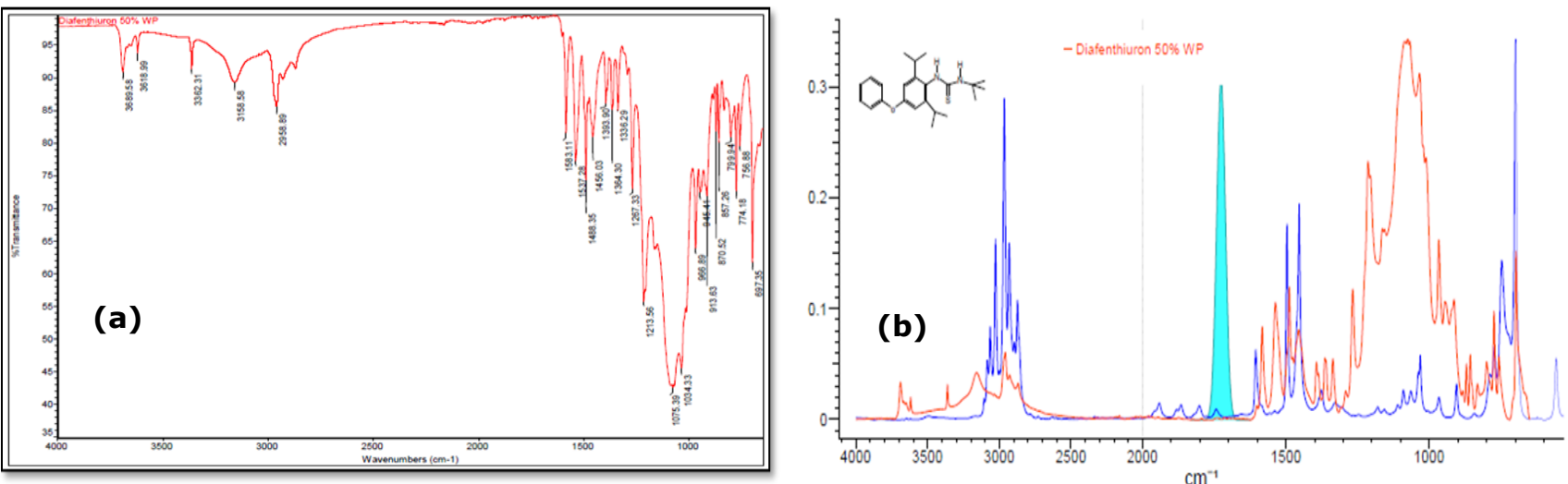

Fig. 16. IR spectra of Diafenthiuron (a). Original spectrum; b). Processed spectrum by KnowitAll).

shown in Fig.16a. In this Fig. 16b, the diafenthiuron spectrum has absorption bands at $3360-3100,1740$ $1715,1300-1160,1200-1050$ due to medium stretching of thioamide bond $\mathrm{N}=\mathrm{H}$, strong stretching of unsaturated ester bond $\mathrm{C}=\mathrm{O}$, strong stretching of unsaturated ester bond $\mathrm{C}-\mathrm{O}$, medium stretching bond $\mathrm{C}=\mathrm{S}$, respectively. The ProcessItIR spectrum showed $100 \%$ intensity at $1075.60 \mathrm{~cm}-1$ and $1034.14 \mathrm{~cm}-1$ has $90.96 \%$. Sample spectra provide the characteristic bands of the active principles additionally than some small bands coming from inert and solvent components of the pesticide formulations.

\section{Conclusion}

The suitability of the vibrational spectrometry for the determination of active ingredients in solid and liquid pesticide formulations at MIR regions indicated signature absorption bands of 1667-1680, 1650-1550, 13401250, 800-580 and830- $600 \mathrm{~cm}^{-1}$, respectively, for $C=0$, $\mathrm{C}-\mathrm{N}=\mathrm{C}, \mathrm{N}-\mathrm{H}, \mathrm{P}=\mathrm{S}$ and halogen bonds. Also, absorption bands of few pesticides were comparable spectra with the available NIST library and Spectrabase. Hence FTIR spectrometry for the direct determination of commercial pesticide formulations can be carried out without any pre-treatment of samples. So the proposed procedure was environmentally friendly for quality control analysis of formulated pesticides. These pesticide molecules provided specific characteristic absorption bands in the mid-IR, located at different wavenumbers providing the qualitative representation of the compounds. This work's principal target has been the advancement of quick and environmentally friendly techniques for the assurance of pesticides in agrochemical definitions utilizing vibrational spectroscopy like FTIR and extending to determine pesticide residue in plant and food material. This can be achieved after calibrating the signature bands for each compound.

\section{ACKNOWLEDGEMENTS}

I express my deep sagacity and gratitude to the Dean, Project Director (COE-SSH) and the Professor \& Head, Dept. of SS\&AC, ADAC\&RI, Trichy for permitting me to carry out this work in the Soil Health Analytical lab (SHAL) of Centre of Excellence in Sustaining Soil Health.

\section{Conflict of interest}

The authors declare that they have no conflict of interest.

\section{REFERENCES}

1. Armenta, S., Quintás, G., Garrigues, S., \& de la Guardia, M. (2004). Determination of cyromazine in pesticide commercial formulations by vibrational spectrometric procedures. Analytica Chimica Acta, 524(1-2), 257-264. doi:https://doi.org/10.1016/j.aca.2004.02.063 
2. Armenta, S., Quintás, G., Garrigues, S., \& de la Guardia, M. (2005a). A validated and fast procedure for FTIR determination of Cypermethrin and Chlorpyrifos. Talanta, 67(3), 634-639. doi:https://doi.org/10.1016/j.talanta.20 05.03.008

3. Armenta, S., Quintás, G., Morales, A., Garrigues, S. \& de la Guardia, M. (2005b). FTIR approaches for diuron determination in commercial pesticide formulations. Journal of Agricultural and Food Chemistry, 53(15), 5842-5847. doi:https://doi.org/10.1021/jf050268f

4. Armenta, S., Garrigues, S. \& de la Guardia, M. (2007). Partial least squares-near infrared determination of pesticides in commercial formulations. Vibrational Spectroscopy, 44(2), 273-278. doi:https://doi.org/10.1016/j.vibsp ec.2006.12.005

5. Armenta, S., Quintas, G., Garrigues, S., \& De la Guardia, M. (2005c). Mid-infrared and Raman spectrometry for quality control of pesticide formulations. TrAC Trends in Analytical Chemistry, 24(8), 772-781. doi:https:// doi.org/10.1016/j.trac.2005.03.017

6. Esler, M. B., Griffith, D. W., Wilson, S. R., \& Steele, L. P. (2000). Precision trace gas analysis by FT-IR spectroscopy. 1. Simultaneous analysis of $\mathrm{CO} 2, \mathrm{CH} 4, \mathrm{~N} 2 \mathrm{O}$, and $\mathrm{CO}$ in air. Analytical Chemistry, 72(1), 206-215. doi:https:// doi.org/10.1021/ac9905625

7. Fenik, J., Tankiewicz, M., \& Biziuk, M. (2011). Properties and determination of pesticides in fruits and vegetables. TrAC Trends in Analytical Chemistry, 30(6), 814-826. doi:https://doi.org/10.1016/j.trac.2011.02.008

8. Lee, L. C., Liong, C.-Y., \& Jemain, A. A. (2017). A contemporary review on Data Preprocessing (DP) practice strategy in ATR-FTIR spectrum. Chemometrics and Intelligent Laboratory Systems, 163, 64-75. doi:https:// doi.org/10.1016/j.chemolab.2017.02.008

9. Moros, J., Armenta, S., Garrigues, S., \&de la Guardia, M. (2006). Quality control of Metamitron in agrochemicals using Fourier transform infrared spectroscopy in the middle and near range. Analytica Chimica Acta, 565(2), 255260. doi:https://doi.org/10.1016/j.aca.2006.02.026

10. Post, E., Rahner, S., Möhler, H. \& Rager, A. (1995). Study of recyclable polymer automobile undercoatings contain- ing PVC using TG/FTIR. Thermochimica acta, 263, 1-6. doi:https://doi.org/10.1016/0040-6031(94)02388-5

11. Qiu, K., Song, X., Lai, Y., Wu, L., Tang, G. \& Min, S. (2013). Comparison of ATR/transmittance FTIR combined with Beer's law and PLS to determine fipronil in matrine formulation. Analytical Methods, 5(18), 4790-4797. doi:https://doi.org/10.1039/C3AY40406D

12. Quintás, G., Armenta, S., Garrigues, S. \& Guardia, M. d. I. (2004a). Fourier transform infrared determination of imidacloprid in pesticide formulations. Journal of the Brazilian Chemical Society, 15(2), 307-312. doi:https:// doi.org/10.1590/S0103-50532004000200023

13. Quintas, G., Armenta, S., Morales-Noe, A., Garrigues, S. $\&$ de la Guardia, M. (2003). Simultaneous determination of Folpet and Metalaxyl in pesticide formulations by flow injection Fourier transform infrared spectrometry. Analytica Chimica Acta, 480(1), 11-21. doi:https://doi.org/10.1 016/S0003-2670(02)01596-9

14. Quintás, G., Morales-Noe, A., Armenta, S., Garrigues, S. \& de la Guardia, M. (2004b). Fourier transform infrared spectrometric determination of Malathion in pesticide formulations. Analytica Chimica Acta, 502(2), 213-220. doi:https://doi.org/10.1016/j.aca.2003.10.044

15. Segal-Rosenheimer, M. \& Dubowski, Y. (2007). Heterogeneous ozonolysis of cypermethrin using real-time monitoring FTIR techniques. The Journal of Physical Chemistry C, 111(31), 11682-11691. doi:https://doi.org/10.1021/ jp072937t

16. Van de Voort, F., Sedman, J., Yaylayan, V., Laurent, C. S. \& Mucciardi, C. (2004). Quantitative determination of moisture in lubricants by Fourier transform infrared spectroscopy. Applied Spectroscopy, 58(2), 193-198. doi:https://doi.org/10.1366\%2F000370204322842922

17. Yang, L., Zhang, X. \& Jiang, L. (2019). Determination of organophosphorus pesticides in fortified tomatoes by fluorescence quenching of cadmium selenium-zinc sulfide quantum dots. Analytical Letters, 52(5), 729-744. doi:https://doi.org/10.1080/00032719.2018.1490311 